\title{
Anti-D Antibodies in Pregnant D Variant Antigen Carriers Initially Typed as RhD+
}

\author{
Jelena Lukacevic Krstic ${ }^{a}$ Slavica Dajak ${ }^{a}$ Jasna Bingulac-Popovic ${ }^{b}$ Vesna Dogic $^{b}$ \\ Jela Mratinovic-Mikulandra ${ }^{a}$

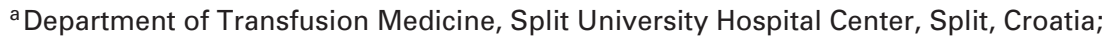 \\ ${ }^{b}$ Croatian Institute of Transfusion Medicine, Zagreb, Croatia
}

\section{Keywords}

Anti-D antibodies - D antigen - D variants .

Pregnant women · HDFN

\section{Summary}

Background: To evaluate the incidence, the consequences, and the prevention strategy of anti-D alloimmunizations of $D$ variant carriers in the obstetric population of Split-Dalmatia County, Croatia. Methods: RhD immunization events were evaluated retrospectively for the period between 1993 and 2012. Women were tested for $\mathrm{RhD}$ antigen and irregular antibodies. Those with anti-D antibody who were not serologically D- were genotyped for RHD. They were evaluated for their obstetric and transfusion history and their titer of anti-D. The neonates were evaluated for RhD status, direct antiglobulin test (DAT), hemoglobin and bilirubin levels, transfusion therapy as well as phototherapy and outcome. Results: Out of 104,884 live births 102,982 women were tested for RhD antigen. Anti-D immunization occurred in 184 women which accounts for $0.9 \%$ of individuals at risk of anti-D formation. 181 cases occurred in women serologically typed as D-. Three women were partial D carriers (DVa $n=2$, DNB $n=1$ ), initially typed $R h D+$, and recognized as $D$ variant carriers after the immunization occurred. Anti-D titer varied from 1:1 to $1: 16$. Six children were RhD+, four had positive DAT, and two underwent phototherapy. Conclusion: Anti-D immunization occurred in pregnant partial D carriers (DVa, DNB). RhD+ children had serologic markers of hemolytic disease of the fetus and newborn (HDFN), with no cases of severe HDFN.

\section{Introduction}

The $\mathrm{RhD}$ antigen is a highly immunogenic antigen significant for obstetric medicine due to the ability of anti-D to cause hemolytic disease of the fetus and newborn (HDFN) [1,2]. Other than regular D+, D antigen could be presented in more than 200 variants currently known as D variants [3]. Reduced expression of the $\mathrm{D}$ antigen is traditionally referred to as 'weak D', while 'partial D' represents variants in which the mutations and gene rearrangements result in the altered protein sequence lacking certain polypeptides on the outer RBC surface [4], although it had been proven that the distinction between weak and partial D is fluid and that weak D types possess slightly to considerably altered D antigens [5]. Most weak D variants in Caucasians include weak D type 1, type 2 and type 3 , which are not known to form alloanti-D [1]. Conversely, partial D carriers are prone to the formation of anti-D. DVI category is the most clinically relevant partial D among Caucasians, being the $\mathrm{D}$ variant most commonly reported to be involved in anti-D immunization as well as HDFN [1]. HDFN in infants from partial D mothers with anti-D has been documented, but in most cases it had been mild, with rare exceptions [6-9].

The low risk of RhD immunization in women with weak D types 1, 2 and 3 is the reason why most authors hold that women with prevalent weak $\mathrm{D}$ types do not require $\mathrm{RhD}$ immunoprophylaxis $[5,10-13]$, and weak D patients could safely receive D+ RBC units [4]. Conversely, women with partial D should be classified as D- considering their prenatal management and RhD immunoprophylaxis $[13,14]$. In Europe, anti-D reagents are selected to deliberately type pregnant DVI carriers as D- to ensure that such mothers would receive $\mathrm{RhD}$ immunoprophylaxis in their second trimester and/or postpartum. The distribution of $\mathrm{D}$ variants varies among different populations, and their recognition depends on the choice of D typing reagents used $[15,16]$.

\section{KARGER}

() 2016 S. Karger GmbH, Freiburg 


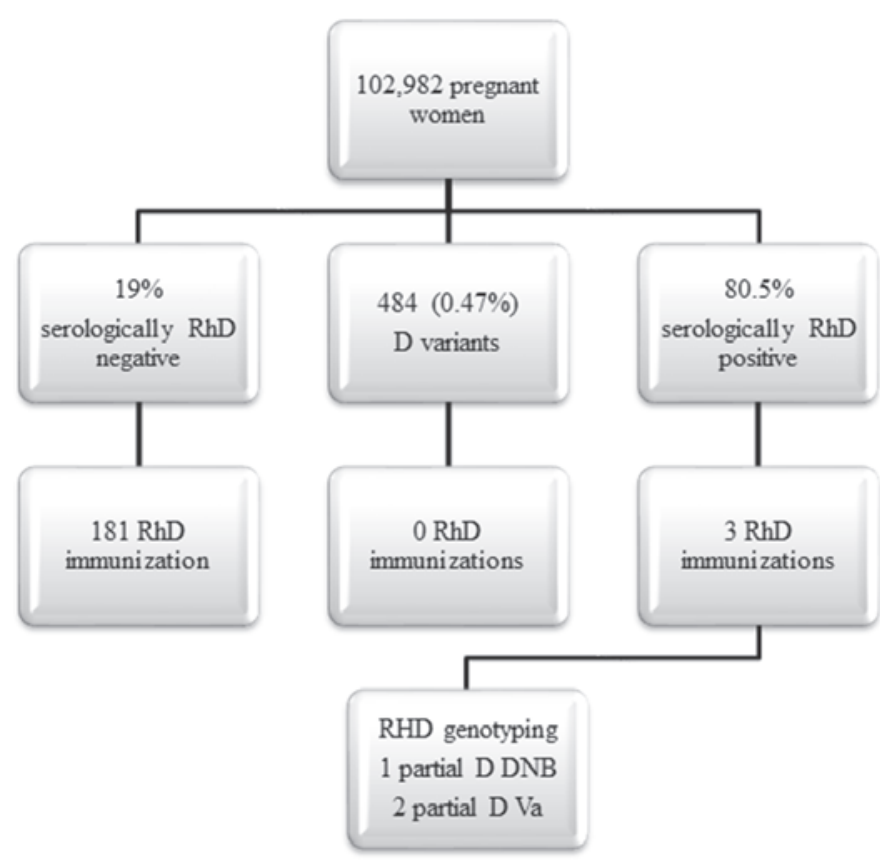

Fig. 1. Results of RhD typing and $\mathrm{RhD}$ immunization events detected in pregnant women between 1993 and 2012. RHD genotyping was done only for the 3 women with anti-D antibodies who were initially typed as D+.

We retrospectively evaluated the cases of $\mathrm{RhD}$ alloimmunization and RhD antigen in pregnant women for a period of 20 years. For women who were not serologically D-, we analyzed the cause of immunization (transfusion or pregnancy), their serologic reactivity, and RHD genotype. Also, we determined whether there had been cases of HDFN among the children of RhD-alloimmunized D variant mothers. The aim of the study was to evaluate the incidence and the consequences of anti-D alloimmunizations of $\mathrm{D}$ variant carriers in the Split-Dalmatia County obstetric population.

\section{Material and Methods}

The present study was performed at the Department of Transfusion Medicine of the Split University Hospital Center, in Split-Dalmatia County, Croatia. SplitDalmatia County has approximately 5,000 births in a year. The Hospital is a regional institution responsible for the overall pregnancy care, monitoring of RBCalloimmunized women, and the management of deliveries. All pregnant women in this region were typed for $\mathrm{ABO}$ and $\mathrm{RhD}$ antigens and tested for irregular antibodies at their initial visit. On each subsequent visit, only indirect antiglobulin test (IAT) control was performed. RhD immunoprophylaxis is routinely administered to $\mathrm{RhD}$ - women after the delivery of $\mathrm{RhD}+$ child. $\mathrm{D}$ variant women were treated as $\mathrm{RhD}$ - if transfusion therapy was required, but they did not receive $\mathrm{RhD}$ immunoprophylaxis. If RHD genotyping revealed partial D antigen, the immunoprophylaxis was administered after the birth of the $\mathrm{RhD}+$ child.

\section{Pregnancies with $D$ Variant and Anti-D}

We retrospectively evaluated $\mathrm{RhD}$ immunization events in the obstetric population for the period of January 1, 1993 to December 31, 2012. In SplitDalmatia County, 104,884 live births were registered between January 1, 1993 and December 31, 2012. During that period, 102,982 pregnancies were tested for $\mathrm{ABO}$ and $\mathrm{RhD}$ antigens and for irregular antibodies at our department $(98 \%$ screening coverage, which is in concordance with a previous study on our obstetric population) [17]. The number of $\mathrm{RhD}$ typing tests and the number of serologically recognized D variants were collected from the manually written protocol for tests of obstetric patients for the period between 1993 and 2001, and from the computer database for the period between 2002 and 2012.

During this period we used immunization tracking forms for the follow-up of pregnancies with anti-D alloantibody, which were filled out after clinically relevant alloantibodies have been detected. From these forms we analyzed the overall number of pregnant women with $\mathrm{RhD}$ immunization, with detailed analysis of RhD-immunized women which were not serologically RhD-. For these women we analyzed immunization events which could cause $\mathrm{RhD}$ immunization, prior pregnancies, prior transfusions, and $\mathrm{RhD}$ immunoprophylaxis administration postpartum; moreover, we analyzed the RHD genotype of D variant women. During the pregnancy, the titer of anti-D antibodies was measured in intervals of 2-4 weeks, and the results for each measurement were recorded in immunization tracking forms. The forms also contained data about the infants' RhD status and direct antiglobulin test (DAT).

Data about $\mathrm{RhD}+$ children from $\mathrm{D}$ variant women with anti-D antibodies were collected from the patients' history on the Department for Pediatrics and Neonatology. Information collected included data about HDFN, neonatal hemoglobin and bilirubin levels, neonatal transfusion therapy and phototherapy, and other neonatal outcomes.

\section{Serologic Typing of RhD Antigen}

In the period from January 1, 1993 to April 10, 2008 routine RhD typing was performed using direct agglutination in tube testing with two out of the following monoclonal reagents: Anti-D M MonoGnost ${ }^{\circledR}$ (MS-201; BioGnost, Zagreb, Croatia); NovaClone ${ }^{\circledR}$ Anti-D IgM+IgG Monoclonal Blend (CI 175-2, D415, 1E4, Immucor Gamma, Dartmouth, NS, Canada); Anti-D MG MonoGnost $^{\circledR}$ (RUM-1 IgM, MS-26 IgG, BioGnost).

If $\mathrm{RhD}$ antigen was typed negative in direct agglutination, it was typed in IAT. RhD antigen typed negative in direct agglutination, but positive in IAT, was classified as 'D variant'. Testing RhD antigen in IAT using column method was performed using Anti-D BioClone ${ }^{\circledR}$ human monoclonal-polyclonal blend (Ortho Clinical Diagnostics, Raritan, NJ, USA).

From April 10, 2008 to December 31, 2012, RhD typing in direct agglutination was performed by automated microcolumn technology using ABO-DD Grouping Cassette (Ortho Clinical Diagnostics) which contains two monoclonal IgM anti-D reagents (clone D7B8, and clone RUM-1). Testing RhD antigen in IAT was omitted after having introduced microcolumn technology. The cases with discrepant serologic results were sent for molecular RHD analysis, and the immunoprophylaxis was implemented for women who were found to be partial D carriers.

\section{RHD Genotyping}

DNA extraction from EDTA blood samples was done manually by QIAamp DNA Blood Mini kit ${ }^{\circledR}$ (Qiaqen, Hilden, Germany) or by Qiacube analyser ${ }^{\circledR}$ (Qiaqen). Molecular typing of D variants was performed by PCR-SSP kits (Ready GeneWeak $D^{\circledR}$ and Ready GeneCDE ${ }^{\circledR}$; Inno-Train, Kronberg im Taunus, Germany), according to the instructions of the manufacturer.

\section{Results}

In the observed period out of 102,982 pregnancies tested for $\mathrm{ABO}$ and $\mathrm{RhD}$ antigens and irregular antibodies, there had been 184 pregnant women affected by RhD alloimmunization in 20,050 women who were $\mathrm{RhD}$ - or $\mathrm{D}$ variant. That accounts for $0.9 \%$ of pregnant women at risk for $\mathrm{RhD}$ alloimmunization. Of those, 181 women were serologically typed $\mathrm{RhD}_{-}$, and 3 immunizations (1.63\% of anti-D immunizations) occurred in women who were carriers of RhD variants. RHD genotyping defined that 2 of 3 women were partial $\mathrm{D}$ type $\mathrm{Va}$, while 1 woman was carrier of partial D type DNB (fig. 1). All 3 women were labeled as $\mathrm{RhD}+$ in their 
Table 1. Causes of RhD immunization in women with partial D

\begin{tabular}{|c|c|c|c|}
\hline \multirow{3}{*}{ Genotype } & \multicolumn{3}{|l|}{ Pregnant women } \\
\hline & 1 & 2 & 3 \\
\hline & DNB category & RHD category Va & RHD category $\mathrm{Va}$ \\
\hline Phenotype & $\mathrm{CcEe}$ & CCee & Ccee \\
\hline Pregnancies & $\begin{array}{l}\text { 1. miscarriage } \\
\text { 2. uneventful } \\
\text { 3. RhD+, DAT 1+ } \\
\text { 4. RhD+, DAT 3+ }\end{array}$ & $\begin{array}{l}\text { 1. miscarriage } \\
\text { 2. RhD+, DAT- } \\
\text { 3. miscarriage } \\
\text { 4. RhD+, DAT- }\end{array}$ & $\begin{array}{l}\text { 1. uneventful } \\
\text { 2. RhD-, DAT- } \\
\text { 3. RhD+, DAT+ } \\
\text { 4. RhD variant, DAT- } \\
\text { 5. RhD-, DAT- } \\
\text { 6. RhD+, DAT+ }\end{array}$ \\
\hline Transfusion & $\begin{array}{l}\text { yes } \\
\text { after 2nd pregnancy* }\end{array}$ & no & $\begin{array}{l}\text { yes } \\
\text { after 1st pregnancy }{ }^{*}\end{array}$ \\
\hline Pregnancy $^{\dagger}$ & $3 \mathrm{rd}$ & 2 nd & $3 \mathrm{rd}$ \\
\hline Cause of immunization & $\begin{array}{l}\text { unclear } \\
\text { (pregnancy or } \\
\text { transfusion) }\end{array}$ & pregnancy & $\begin{array}{l}\text { unclear } \\
\text { (pregnancy or } \\
\text { transfusion) }\end{array}$ \\
\hline
\end{tabular}

*Women received RhD positive RBCs.

Pregnancy in which anti-D antibody was detected.

Table 2. Neonatal outcomes in $\mathrm{RhD}+$ children in mothers with partial $\mathrm{D}$ and anti-D immunization

\begin{tabular}{|c|c|c|c|c|c|c|c|}
\hline \multirow[t]{2}{*}{ Mother } & \multirow[t]{2}{*}{ Gravida } & \multirow[t]{2}{*}{ Titer anti-D* } & \multicolumn{5}{|c|}{ Newborn } \\
\hline & & & $\mathrm{DAT}^{\dagger}$ & hemoglobin, $\mathrm{g} / \mathrm{l}^{ \pm}$ & bilirubin, $\mu \mathrm{mol} / \mathrm{l}^{\ddagger}$ & phototherapy & top-up transfusion \\
\hline \multicolumn{8}{|l|}{1} \\
\hline Partial D & 3 & $<1$ & $1+$ & 180 (day 1$)$ & 179 (day 5) & yes & no \\
\hline $\mathrm{DNB}$ & 4 & $1: 2$ & $4+$ & 177 (day 8) & 234 (day 7) & no & yes $^{\S}$ \\
\hline \multicolumn{8}{|l|}{2} \\
\hline Partial D & 2 & $<1$ & - & 196 & 98 & no & no \\
\hline Type Va & 4 & $<1$ & - & 162 (day 2) & 258 (day 1 ) & yes & no \\
\hline \multicolumn{8}{|l|}{3} \\
\hline Partial D & 3 & $1: 16$ & $1+$ & 186 & 100 & no & no \\
\hline Type Va & 6 & $1: 1$ & $1+$ & 192 & 105 & no & no \\
\hline
\end{tabular}

*The titer of anti-D antibodies at the end of pregnancy. The titer was performed by tube method.

'The DAT of the newborn was performed by microcolumn method in polyspecific AHG cassette (Ortho Clinical Diagnostics, Raritan, New Jersey, USA).

${ }^{ \pm}$The lowest hemoglobin level during the hospital stay, measured from venous blood. Reference interval: 145-225 g/l.

†The highest total bilirubin level during the hospital stay. Reference interval for term newborns: $0-1$ day $<103 \mu \mathrm{mol} / 1 ; 1-2$ days $<137 \mu \mathrm{mol} / 1 ; 2-5$ days $<205$

$\mu \mathrm{mol} / 1$; older newborns 3.4-17.1 $\mu \mathrm{mol} / \mathrm{l}$.

${ }^{s}$ The transfusion was performed due to the surgical procedure.

initial serology typing of $\mathrm{RhD}$ antigen, due to the choice of reagents which were used at the time (Anti-D M MonoGnost, NovaClone Anti-D IgM+IgG Monoclonal Blend. Therefore, 2 out of 3 received $\mathrm{RhD}+\mathrm{RBCs}$, and neither of them received antenatal or postnatal anti-D immunoprophylaxis. The characteristics of partial D woman with anti-D immunization are shown in table 1 . One of them (DVa) was immunized exclusively due to pregnancy, while for other the 2 women (DVa and DNB) the immunization was most likely the consequence of being transfused with $\mathrm{RhD}+\mathrm{RBCs}$ (table 1).

During the study period, 484 women were typed as D variant (Du) using serologic typing methods only. These women were not given immunoprophylaxis, but they received $\mathrm{D}-\mathrm{RBC}$ units in cases of transfusion (fig. 1). There were no cases of anti-D formation among them.

Table 2 shows data on anti-D titer of the immunized mothers as well as data on their affected children, including neonatal DAT, hemoglobin, bilirubin, phototherapy, and transfusion therapy.

The DNB variant woman was found to be immunized during the investigation of the newborn's positive DAT. Since the woman was the citizen of Bosnia and Herzegovina and had arrived to Croatia shortly prior to her labor, no data were available of her earlier immunohematology testing. However, using information on her health history, we found that she had received A+ RBCs after pre- 
vious baby's delivery. The newborn required only phototherapy. Her subsequent pregnancy resulted in the birth of a child whose DAT was strongly positive and the baby required top-up transfusion in his 8th day of life. However, the transfusion was not attributed to hemolysis, but to surgery due to the incarcerated hernia.

The second woman (partial D type Va) was found to be alloimmunized on her repeated routine screening for irregular antibodies in the 34th week of her second pregnancy. Her transfusion history was negative. Although she was carrying an $\mathrm{RhD}+$ child, her anti-D titer remained very low throughout the pregnancy. The newborn's DAT after delivery was negative, and the levels of bilirubin and hemoglobin were within the reference ranges. However, her second $\mathrm{RhD}+$ child had pathologically high bilirubin levels and required phototherapy.

The third woman (partial D type Va) was found to be alloimmunized on the routine blood grouping and screening in the second pregnancy, her first pregnancy was uneventful but followed by transfusion of two units of $\mathrm{A}+\mathrm{RhD}+\mathrm{RBCs}$. Despite the fact that out of her four subsequent pregnancies, two resulted in birth of $\mathrm{RhD}+$ children whose DAT was $1+$ positive and that she had the highest anti-D titer of all 3 women, no pathologically high bilirubin levels, visible jaundice, or anemia were detected in either of her children, their postnatal periods were uneventful, and they were discharged from hospital without complications. Overall, the women with partial D variants and anti-D immunization in total had six pregnancies involving $\mathrm{RhD}+$ children. Two infants needed phototherapy, but there had been no cases of severe HDFN.

\section{Discussion}

This study analyzes $\mathrm{RhD}$ immunizations in pregnant women which were carriers of D variants, in Split-Dalmatia County. We found 184 pregnant women with anti-D antibodies, which accounts for $0.9 \%$ of individuals at risk of anti-D formation. This is in concordance with a recent work on our obstetric population with anti-D [18]. Such a high percentage is attributed to the fact that antenatal prophylaxis is not implemented in our country, and the need for it to become standard practice has already been stressed upon [18]. 181 alloimunized women were serologically typed as $\mathrm{RhD}-$. Three alloimmunized persons initially typed as $\mathrm{RhD}+$ were partial D carriers. There were no cases of anti-D formation among pregnant women which were initially recognized as $\mathrm{D}$ variant and were assigned 'Du' status.

In the guidelines of some countries for prevention of anti-D alloimmunization, it is stated that weak D or Du women do not require prophylaxis [19] while newer recommendations stress out the importance of resolving unclear $\mathrm{RhD}$ typing results [20], and treatin all D variants other than weak D types 1, 2 and 3 as D- [21].

Until 2008, RhD antigen testing in our center was based on serology only. Women found to be $\mathrm{RhD}$ - using tube method by direct agglutination but $\mathrm{RhD}+$ in indirect agglutination were assigned $\mathrm{D}$ variant or $\mathrm{Du}$, and did not receive $\mathrm{RhD}$ immunoprophylaxis; however, they were regarded as $\mathrm{RhD}$ - considering transfu- sion therapy. Using this approach, pregnant women with partial D antigen were classified as D variant or Du. and they were not given immunoprophylaxis; consequently, the immunization events due to pregnancy were not prevented. In our center since 2008, cases with unclear results of serologic $\mathrm{RhD}$ typing underwent molecular RHD analysis, and the immunoprophylaxis was implemented for women who were genotyped as partial D carriers. Other studies also point out the importance of RHD genotyping in pregnant women with $\mathrm{D}$ variant antigen in order to correctly identify partial D carriers which need to be administered immunoprophylaxis [12, 20].

All 3 alloimmunized partial D women in our research did not receive immunoprophylaxis, and 2 of them received $\mathrm{RhD}+$ units of RBCs, because they were not initially recognized to be carriers of $\mathrm{D}$ variants, and were typed as $\mathrm{RhD}+$. Only 1 immunization event in a partial D woman was linked entirely to pregnancy. For the remaining $2 \mathrm{RhD}$ immunizations it was not possible to determine whether transfusion or pregnancy caused immunization. This suggests that recognizing $\mathrm{D}$ variants during serologic typing is essential to ensure that pregnant partial $\mathrm{D}$ carriers are treated as $\mathrm{RhD}$ - considering transfusion therapy and immunoprophylaxis.

After RhD typing in IAT was discarded for pregnant women in 2008, those with DVI variant, if present in our obstetric population, were typed as $\mathrm{RhD}$ - while previously they were assigned as $\mathrm{D}$ variant or $\mathrm{Du}$, since DVI gives positive reactions in indirect agglutination with anti-D reagent used for IAT typing. Still, we found no cases of anti-D in DVI mothers. Despite DVI variant being held for the most clinically relevant partial D variant among Caucasians [1], the affected women in our study were of DVa and DNB categories. Moreover, the research performed on blood donors from the same geographic region found no carriers of partial DVI category [22]. $\mathrm{DNB}$ is known to be the most prevalent partial $\mathrm{D}$ variant in the neighboring regions of Central Europe [23]. In our case, the only DNB carrier originated from a neighboring geographic region of Bosnia and Herzegovina while both DVa carriers originated from the investigated area of Split-Dalmatia County. This implies the necessity to take into account the geographic variability in the prevalence of $\mathrm{D}$ variants while implementing $\mathrm{RhD}$ typing and immunoprophylaxis standards.

No cases of anti-D formation in women which were initially assigned $\mathrm{RhD}+$ or Du status were attributed to weak D types when genotyping was performed on the recorded alloimmunized cases. This was expected since the most prevalent weak D variants prevalent in Croatia are weak D type 3, type 1 and type 2 while in the Mediterranean part of Croatia, in which the study was performed, the most common is weak D type 1, followed by type 3 and 2 [24]. Opposite to the findings of authors in non-Caucasian populations, with weak $\mathrm{D}$ types prone to anti-D formation being common, it is safe to assume that weak $\mathrm{D}$ carriers in our obstetric population do not require immunoprophylaxis [25]. However, since in a previously published study [24] on Croatian population weak D type 4.2 , which is known to make anti- $\mathrm{D}$, was found to be present in the Mediterranean part of Croatia, caution is mandatory. Moreover, in a study on RhD- pregnant women by Hyland et al. [26], D variant 
carriers were found among alloimmunized women. Some partial D RBCs fail to react with anti-D even with IAT [27]. In our study genotyping was not performed in alloimmunized RhD- women; therefore, some D variant individuals with anti-D might have been missed by our serologic techniques used at the time.

Similarly to the findings of most other authors, anti-D immunizations of partial D women resulted mostly in serologic markers of HDFN, but without serious clinical consequences $[6,7,9]$. It is interesting to notice that in our study the woman with the highest anti-D titer of all 3 women gave birth to children whose DAT was only $1+$, while the children of the woman with lower titer were more prominently serologically affected, with DAT $4+$. The factors involving severity of HDFN are still a subject of potential research $[28,29]$. Despite most cases of HDFN in partial D mothers being mild, fatal outcome has been also reported [8].

While anti-D formation in non-RhD- women is extremely rare, the demonstrated immunization events confirm that it is relevant to correctly identify partial D carriers, which require to be regarded as $\mathrm{RhD}$ - considering transfusion therapy and immunoprophylaxis. Recent research from USA found that performing RHD genotyping among pregnant women with serologically weak D phenotypes is a cost-neutral strategy, with cost savings likely to increase slightly over time [30]. Along with undisputed importance of RHD genotyping in resolving discrepant $\mathrm{RhD}$ typing results and discerning $\mathrm{D}$ variants, it is necessary to stress out the relevance of serologic typing, since the need for genotyping will not be recognized if $\mathrm{D}$ variants are not recognized during the initial serologic typing and $\mathrm{D}$ variant carriers are assigned $\mathrm{RhD}+$. In our study, all 3 pregnant women with anti-D were not initially recognized as $\mathrm{D}$ variants due to the strong reactivity of their RBCs with monoclonal reagents used at the time. We can conclude that the choice of reagents for serologic $\mathrm{RhD}$ typing is crucial for the recognition of $\mathrm{D}$ variants, and consequently, the recognition of the need for molecular $\mathrm{RhD}$ typing.

Finally, in this study we found that there had not been RhD immunization cases in pregnant women initially typed as $\mathrm{D}$ variant, but the immunizations did occur in women which were typed as $\mathrm{RhD}+$. This implies the need for further investigation in order to make appropriate choice of reagents for serologic $\mathrm{RhD}$ typing which will detect $\mathrm{D}$ variants at risk of anti-D alloimmunization in our population.

The limitations of this retrospective study are long tracking period, changes in methods and reagents that were used during the study period as well as the testing algorithm. The changes include omitting RhD typing in IAT after 2008 and introduction of immunoprophylaxis for pregnant women with partial D antigen after 2008, while before 2008 prophylaxis was not administered to women with any D variant. Another limitation of our study is the fact that genotyping was not performed in alloimmunized $\mathrm{RhD}$ - women.

Despite this limitations, we believe it is very important to follow up the obstetric population through long period of time. This would enable us to assess the adequacy of RhD antigen serologic typing strategy and immunoprophylaxis administration in pregnant women with $\mathrm{D}$ variants.

\section{Acknowledgments}

The authors are grateful to Pera Erceg-Maglic, Hanija Dadic-Jadric, Branka Skelin, Dejana Bogdanic, Ivica Bradaric, Vedrana Burilovic and Nina Ipavec for collecting data during research.

\section{Ethical Prerequisites}

The study was approved by the Ethical Committee of the Split University Hospital Center.

\section{Disclosure Statement}

Authors disclose no conflict of interest.

\section{References}

1 Mollison PL, Engelfriet CP, Contreras M: Blood Transfusion in Clinical Medicine, Oxford, Blackwell, 2006 pp163-208;498-525.

2 Urbaniak SI, Greiss MA: RhD haemolytic disease of the fetus and the newborn. Blood Rev 2000;14:44-61.

3 International Society of Blood Transfusion: Red Cell Immunogenetics and Blood Group Terminology. www isbtweb.org/working-parties/red-cell-immunogeneticsand-blood-group-terminology/ (last accessed September 28, 2016)

4 Wagner FF, Gassner C, Müller TH, Schönitzer D, Schunter F, Flegel WA: Molecular basis of weak D phenotypes. Blood 1999;93:385-393.

$\checkmark 5$ Wagner FF, Frohmajer A, Ladewig B, Eicher NI, Lonicer CB, Muller TH, Siegel Flegel WA: Weak D alleles express distinct phenotypes. Blood 2000;95:2699_ 2708.

6 Prasad MR, Krugh D, Rossi KQ, O’Shaughnessy RW: Anti-D in Rh positive pregnancies. Am J Obstet Gynecol 2006;195:1158-1162.
7 Gardener GJ, Legler TJ, Hyett JA, Liew YW, Flower RL, Hyland CA: Anti-D in pregnant women with the RHD(IVS3+1G $>$ A)-associated DEL phenotype. Transfusion 2012;52:2016-2019.

8 Cannon M, Pierce R, Taber EB, Schucker J: Fatal hydrops fetalis caused by anti-D in a mother with partial D. Obstet Gynecol 2003;102:1143-1145.

9 Jakobsen MA, Nielsen C, Sprogøe U: A case of high-titer anti-D hemolytic disease of the newborn in which late onset and mild course is associated with the D variant, RHD-CE(9)-D. Transfusion 2014;54:24632467.

10 Wang D, Lane C, Quillen K: Prevalence of RhD variants, confirmed by molecular genotyping, in a multiethnic prenatal population, Am J Clin Pathol 2010;134: 438-442.

11 Flegel WA: How I manage donors and patients with a weak D phenotype. Curr Opin Hematol 2006;13:476483
12 Sandler SG, Flegel WA, Westhoff CM, Denomme GA, Delaney M, Keller MA, Johnson ST, Katz L, Queenan JT, Vassallo RR, Simon CD; College of American Pathologists Transfusion Medicine Resource Committee Work Group: It's time to phase in RHD genotyping for patients with a serologic weak D phenotype. Transfusion 2015;55:680-689.

13 Minon JM, Gerard CH, Chantraine F, Nisolle M: AntiD prophylaxis reviewed in the era of foetal RHD genotyping. J Blood Disord Transfus 2015;6:302.

14 Lubusky M: Prevention of RhD alloimmunization in $\mathrm{RhD}$ negative women. Biomed Pap Med Fac Univ Palacky Olomouc Czech Repub 2010;154:3-8.

15 Avent ND, Reid ME: The Rh blood group system: a review. Blood 2000;95:375-387.

16 Christiansen M, Samuelsen B, Christiansen L, Morbjerg T, Bredahl C, Grunnet N: Correlation between serology and genetics of weak D types in Denmark. Transfusion 2008;48:187-193. 
17 Dajak S, Stefanovic V, Capkun V: Severe hemolytic disease of fetus and newborn caused by red blood cel antibodies undetected at first-trimester screening. Transfusion 2011;51:1380-1388.

18 Dajak S, Roje D, Hašpl ŽH, Maglić PE: The importance of antenatal prevention of $\mathrm{RhD}$ immunisation in the first pregnancy. Blood Transfus 2014;12:410-415.

19 Fung Kee Fung K, Eason E: Prevention of RhD alloimmunization. J Obstet Gynaecol Can 2003;25:765-773.

20 Qureshi H, Massey E, Kirwan D, Davies T, Robson S, White J, Jones J, Allard S: BCSH guideline for the use of anti-D immunoglobulin for the prevention of haemolytic disease of the fetus and newborn. Transfus Med 2014;24:8-20.

21 Bennardello F, Coluzzi S, Curciarello G, Todros T, Villa S; Italian Society of Transfusion Medicine and Immunohaematology (SIMTI) and Italian Society of Gynaecology and Obstetrics (SIGO) working group Recommendations for the prevention and treatment of haemolytic disease of the foetus and newborn. Blood Transfus 2015;13:109-134.
22 Dajak S, Lukacevic Krstic J, Körmöczi G, Dogic V, Burilovic V: Characteristics and frequency of DEL phenotype detected by indirect antiglobulin test in Dalmatia county of Croatia. Transfus Apher Sci 2014; 50:210-213.

23 Wagner FF, Eicher NI, Jørgensen JR, Lonicer CB, Flegel WA. DNB: a partial D with anti-D frequent in Central Europe. Blood 2002;100:2253-2256.

24 Dogic V, Bingulac-Popovic J, Babic I, Hundric-Haspl Z, Jurakovic-Loncar N, Mratinovic-Mikulandra J, Vuk T,Balija M, Jukic I: Distribution of weak D types in the Croatian population. Transfus Med 2011;21:278-279.

25 Abdelrazik AM, Elshafie SM, Ezzat Ahmed GM, Abdelaziz HM: Combining serology and molecular typing of weak D role in improving D typing strategy in Egypt. Transfusion 2013;53(suppl 2):2940-2944.

26 Hyland CA, Gardener GJ, O’Brien H, Millard G, Gibbons K, Tremellen A, Ochoa-Garay G, Flower RL, Hyett JA: Strategy for managing maternal variant RHD alleles in Rhesus D negative obstetric populations during fetal RHD genotyping. Prenat Diagn 2014;34:56-62.
27 Roubinet F, Apoil PA, Blancher A: Frequency of partial D phenotypes in the south western region of France. Transfus Clin Biol 1996;3:247-255.

28 Hadley AG: Laboratory assays for predicting the severity of haemolytic disease of the fetus and newborn. Transpl Immunol 2002;10:191-198.

29 Hadley AG: A comparison of in vitro tests for predicting the severity of haemolytic disease of the fetus and newborn. Vox Sang 1998;74(suppl 2):375-383.

0 Kacker S, Vassallo R, Keller MA, Westhoff CM, Frick KD, Sandler SG, Tobian AA: Financial implications of RHD genotyping of pregnant women with a serologic weak D phenotype. Transfusion 2015;55:2095-2103. 